histological inflammatory response and scored according to the Amniotic Fluid Infection Nosology Committee of Perinatal Section, the Society for Pediatric Pathology, and reported by Redline et al. in 2003.

Results Chorioamnionitis was seen in 42/101(41.6\%) placentae and this occurred significantly more often in newborn babies with probable or proven EONS $(\mathrm{p}<0.05)$. No features of chorioamnionitis were seen in 51/61(83.6\%) of newborn babies with suspected EONS $(\mathrm{p}<0.05)$.

Conclusions Histological examination of the placenta for acute chorioamnionitis has statistically significant correlation with probable and proven EONS and can be a potential tool to guide clinical decision-making and thereby antibiotic use if report is made available within 24 hours as in frozen section study.

\section{POST-OPERATIVE PAEDIATRIC INTRAVENOUS MAINTENANCE FLUIDS AUDITED AGAINST NATIONAL GUIDANCE}

Rachel Gunnell, Rebecca Prince. United Kingdom

\subsection{6/bmjpo-2021-RCPCH.17}

Background In 2015, the National Institute for Health and Care Excellence (NICE) published guidance on paediatric intravenous (IV) fluids, NG29 'Intravenous fluid therapy in children and young people in hospital'. This was in response to a statement by the National Patient Safety Agency (NPSA) regarding the risk of hyponatraemia.

Objectives This project aimed to determine healthcare professional's adherence to NG29 in post-operative paediatric patients, receiving at least 24 hours of IV maintenance fluids in the Leeds Children's Hospital.

Methods The audit took place between October 2019 and March 2020 in Leeds Children's Hospital. Data was collected over three separate periods, totalling 35 days, following a prospective cohort study design. Patients were followed up for 72 hours from the end of their surgery or until they stopped fluids (if this occurred sooner). Neonates, infants weighing less than three kilograms, and young people over the age of 16 were excluded. The prescribing of fluids, and the monitoring of electrolytes, blood glucose and fluid balance, were audited against NG29.

Results Of a total of 59 patients, 42 received fluids for more than 24 hours (17 males and 25 females). Age ranged from one month to 15 years and weight ranged from $3.61 \mathrm{~kg}$ to $92.00 \mathrm{~kg}$. $62 \%$ of surgeries were elective and $76 \%$ were major. Hypotonic fluids were not prescribed for any of the patients, but only $33 \%$ and $10 \%$ met the guidance for monitoring urea and electrolytes and glucose, respectively. Fluid balance charts were in regular use for all patients, however not all of them fully complied with NICE guidance and only $17 \%$ had complete fluid balance charts. Overall, only $12 \%$ of patients met the NICE guidance.

Conclusions Considering the danger of prescribing children hypotonic fluids, their discontinuation is encouraging. However, the lack of recommended monitoring means that patients could receive fluids that are not appropriate, and poor-quality fluid balance charts may make it difficult for prescribers to assess a patient's fluid status. These results suggest a need for change in practice in Leeds to meet NICE guidance and a possible need for other organisations to audit their practice.

\section{EARLY RECOGNITION OF PAEDIATRIC INFLAMMATORY MULTISYSTEM SYNDROME TEMPORALLY ASSOCIATED WITH SARS-COV-2 (PIMS-TS) - A CASE SERIES}

Nicola Scanlon, Nicola Scanlon, James Hibberd, Fionnghuala Fuller. United Kingdom

\subsection{6/bmjpo-2021-RCPCH.18}

Background PIMS-TS has emerged as a novel disease entity. A recent systematic review reported on 662 patients diagnosed with PIMS-TS. Most of these previous studies have focused on children's presentation at the time of admission to tertiary centres and the subsequent investigations and outcomes.

Objectives This case series aims to analyse the clinical features of PIMS-TS at initial presentation and admission to explore if there are any early features of disease, which could enable earlier identification of these patients.

Methods Retrospective data was analysed for all children (aged 0-16 years) seen in North Middlesex University Hospital Paediatric Emergency Department (PED) between March and May 2020 with an eventual discharge diagnosis of 'PIMS-TS' or who fulfilled the RCPCH case definition criteria for PIMS-TS. 11 patients met the criteria for inclusion. Data was collected based on the RCPCH guideline including patient demographics, presenting symptoms and laboratory findings.

Results The mean age at presentation was 7.8 years (Range 18 months -13 years). Six patients were 're-presentations'. The mean time interval between first presentation and eventual admission was 2.7 days (Range 0 - 5 days). The most common symptoms at first presentation were: fever (100\%), vomiting (67\%), headache $(50 \%)$ and rash $(50 \%)$. Two patients were ambulated on IV antibiotics whilst the remaining four patients were reassured and discharged from PED with no follow up. All three patients who had bloods tested on first presentation had a raised CRP and lymphopenia. Two patients had hyponatraemia (67\%).

The most common presenting symptoms at admission were fever (100\%), abdominal pain (73\%), rash $(55 \%)$, vomiting $(55 \%)$ and difficulty in breathing (55\%). Four patients $(36 \%)$ were hypotensive on initial assessment. The pathology results showed that nearly all patients had a raised CRP (91\%) and five patients were lymphopenic (45\%). The mean CRP was markedly elevated $(212 \mathrm{mg} / \mathrm{L})$. ESR was measured in two patients and was raised in both. Eight patients were hyponatraemic (73\%). Fibrinogen, D Dimer and fibrinogen results were elevated in all cases where these were measured. Swab results for SARS-COV-2 PCR were negative in all 11 cases. Five patients tested positive for SARS-COV-2 antibody tests. Kawasaki symptoms were underrepresented in this case series with no such symptoms at first presentation and only some such as conjunctivitis (27\%) and mucous membrane involvement (9\%) appearing in a small number at admission.

Conclusions The results of this case series suggest that children with early PIMS-TS can present to PED with a non-specific febrile illness a few days before they become unwell with the more severe later features. There is a suggestion that certain abnormal blood test results at this point may indicate early PIMS-TS. Further research is needed to ascertain the significance of these findings. The authors support routine nationalised data collection for all cases of PIMS-TS. In addition to exploring optimal treatment options, a review of early signs, symptoms and investigation results will assist in answering the question of whether current accepted practices for 